\title{
Afectividad en adolescentes anoréxicas y bulímicas evaluadas con el psicodiagnóstico de Rorschach
}

Gisella La Madrid

Universidad de Lima

Recibido: 17 de marzo del 2011 / Aprobado: 5 de mayo del 2011

Se estudiaron las características del componente afectivo en una muestra de adolescentes diagnosticadas con anorexia $(n=18)$ y bulimia $(n=17)$. Para la medición de dicha variable se utilizó el psicodiagnóstico de Rorschach y los hallazgos se analizaron a la luz del sistema comprehensivo del Rorschach (Exner, 2007). Puesto que no existían diferencias significativas entre la afectividad de las adolescentes anoréxicas y bulímicas, se decidió integrar ambos grupos, habiéndose finalmente estudiado la afectividad de las jóvenes con trastornos alimentarios. Los resultados se discuten en función del desarrollo evolutivo en la adolescencia, analizándose además la influencia de factores intra-e interpersonales en la conformación de los trastornos alimentarios.

adolescencia / anorexia / bulimia / afectividad / psicodiagnóstico de Rorschach

Affectivity in anorectic and bulimic adolescents evaluated with Rorschach's Psychodiagnostics

The present study discusses the characteristics of the affective component in a sample of adolescents diagnosed with anorexia $(n=18)$ and bulimia $(n=17)$. To measure this variable we used the Rorschach Psychodiagnostics and the findings were analyzed using the Rorscharch's Comprehensive System (Exner, 2007).

Since there were no significant differences between the affectivity of anorectic and bulimic adolescents, both groups were integrated and thus the study was focused on the total group with eating disorders. Results are discussed in terms of evolutionary development in adolescence. The influence of intrapersonal and interpersonal factors in shaping eating disorders is also analyzed.

adolescence / anorexia / bulimia /affectivity / Rorschach psychodiagnosis

Correo electrónico: gisellalamadrid@gmail.com 
Con la llegada de la adolescencia aparecen nuevas tareas de desarrollo que las jóvenes deben aprender a dominar (Horrocks, 1986). Uno de los grandes retos viene desde lo biológico, pues los cambios en la apariencia física amenazarán la imagen corporal hasta entonces constituida forzando a la joven a aceptar la nueva madurez genital, reevaluar su nuevo aspecto externo así como adaptarse a sus nuevas sensaciones y necesidades (Erikson, 1979; Muuss, 1974).

Si bien esta transición resulta difícil por sí misma, en la actualidad, el problema se complejiza ya que tanto la corporalidad como el psiquismo adolescente deberán asentarse dentro de un medio sociocultural que impone ciertos modelos de belleza. En ese sentido, la actual sociedad occidental idolatra los cuerpos modélicos, entendidos estos como tubulares, andróginos y sin curvas. Las figuras esbeltas representan belleza, juventud, éxito y poder; entonces, la delgadez se convierte en un ideal que la mayoría de mujeres desea alcanzar (Hevia, 2003; Kogan, 2003).

A pesar de que la cultura es un referente importante, la familia y los pares constituyen también figuras clave en la construcción de la identidad (Polivy \& Herman, 2002). Los padres ayudan a moldear la personalidad de los hijos y si bien su influencia se reduce progresivamente, no dejan de constituir una base segura a la cual volver en caso de dificultades (Papalia, Olds \& Feldman, 2009).
No obstante, la llegada de la adolescencia puede percibirse como amenazante, especialmente para aquellas familias que desde ya contaban con un precario equilibrio. Entornos excesivamente rígidos o con fronteras difusas inhiben el adecuado desarrollo de la joven. Asimismo, familias que niegan las dificultades, desalientan la comunicación eficaz y son intrusivas o permisivas en exceso, generan un apego inseguro en los hijos, impiden el desarrollo de estrategias de afrontamiento eficaces y crean dificultades para responder en forma adecuada a las necesidades emocionales (Polivy \& Herman, 2002; Raich, 1998; Rausch, 1996). Finalmente, entornos que valoran a sus miembros principalmente por su apariencia originarán una baja autoestima que facilitará el descontento con la imagen corporal (Polivy \& Herman, 2002; Rausch, 1996).

Por otro lado, el grupo de pares constituye un modelo social que si bien brinda a la joven un sentido de pertenencia y ayudan en la construcción de una identidad social, también pueden, a través de comentarios, actitudes y comportamientos, influir sobre el grado de satisfacción con el propio cuerpo (Raich, 2001).

Dada la gran presión sociocultural por lograr un cuerpo delgado, no resulta extraño que surja un elevado descontento con la propia apariencia. Se abre entonces el campo para diversas patologías, entre ellas la anorexia y la 
bulimia nerviosa. No obstante, además de la influencia externa, se necesita la convergencia de ciertos factores de riesgo individuales que propicien el inicio y mantenimiento de estos trastornos alimenticios.

De esta manera, destaca que el haber sufrido de abuso, traumas o acoso escolar durante la niñez probablemente determina dificultades posteriores para distinguir y expresar emociones y sensaciones; condición relacionada con el desarrollo de trastornos alimenticios en la adolescencia (Polivy \& Herman, 2002). Dentro de la esfera afectiva, el haber construido una baja autoestima, presentar ánimo depresivo, ansiedad generalizada o tendencia a la irritabilidad, constituyen factores de riesgo importantes. De igual forma, el experimentar sentimientos displacenteros sobre el cuerpo aumentaría la preocupación por este (Raich, 1998).

Así, se observa que los desórdenes en la alimentación responden a un origen multicausal en el que se necesita la conjunción de varios factores, desde socioculturales hasta rasgos de personalidad, para que estos se puedan instaurar (Polivy \& Herman, 2002; Rausch, 1996).

\section{EL SENTIR DE LA ADOLESCENTE}

La aparición repentina de la crisis puberal pone en peligro la anterior confianza en el cuerpo (Wetzel, en Reusche, 1999). Indudablemente esto producirá fuertes alteraciones en el equilibrio afectivo que obligarán a la nueva adolescente a reevaluar tanto su mundo interno como su vinculación con el entorno (Erikson, 1979).

De esta manera, dado que las presiones son muchas y el yo aún es débil es normal que aparezca una gran inestabilidad afectiva, caracterizada por el rápido cambio en los estados de ánimo, además de una experiencia más intensa de las emociones. No resulta extraño entonces que las adolescentes promedio experimenten afectos disfóricos como tristeza, insatisfacción, angustia, irritabilidad y/o malestar afectivo en algún momento. Además, puesto que se trata de un periodo de transición psicológica, la identidad y la autoestima se verán amenazadas y es probable que también la ansiedad se eleve (Freud, 1971).

Ante tal movilización interna, el yo pondrá en marcha mecanismos que le permitan defenderse. Entre ellos, destacan los identificados por Anna Freud (1971), quien reconoce el uso de la intelectualización y de actitudes ascéticas para contrarrestar los impulsos. Por supuesto, estos recursos no son siempre efectivos por lo que en ciertas ocasiones se esperan conductas impulsivas o descargas afectivas poco moduladas.

De otro lado, si bien la adolescente puede aislarse momentáneamente del medio para darle paso a la introspección, también es cierto que las habilidades sociales mejoran, permitiéndole 
vincularse con sus pares de manera más efectiva. En ese sentido, el aumento de las interacciones será favorable pues no solo pone en evidencia a un yo capaz de relacionarse, sino que además será dentro de este círculo social que la joven desarrollará mayor intimidad y expresión emocional (Horrocks, 1986; Papalia et al., 2009).

\section{LOS AFECTOS EN LA ADOLESCENTE \\ ANORÉXICA Y BULÍMICA}

La anorexia y la bulimia son consideradas trastornos de la conducta alimentaria (TCA) dado el patrón anormal de ingestas. En ambos casos, las jóvenes rechazan, de manera contundente, el mantener un peso esperado para su edad y talla; presentan temor intenso a engordar así como una alta distorsión en la imagen corporal. Como resultado, utilizan diversos métodos que, suponen, les ayudarán a bajar de peso. El uso de la restricción alimentaria es más común en los casos de anorexia, aunque existe una variante en la que la disminuida ingesta se acompaña de purgas. En la bulimia, en cambio, las jóvenes son más propensas a compensar la sobreingesta con purgas (vómitos, laxantes, diuréticos, enemas u otros fármacos) o conductas compensatorias (ejercicios excesivos o ayuno) (Asociación Psiquiátrica Americana [APA], 2002). Sin embargo, dichos síntomas constituyen solo una parte de un conflicto más complejo, pues aunque parezcan solo desórde- nes asociados a la comida, en ambos casos subyace un trasfondo emocional (Zusman, 2009; Kirzman, 2002).

Barbarich, Kaye y Jimerson (2003) señalan que la falta de nutrientes genera cambios en el estado de ánimo de las adolescentes anoréxicas, primando la experiencia de afectos disfóricos, especialmente depresión y ansiedad. Por otro lado, disminuye la expresión emocional y se acrecientan las dificultades para reconocer aquello que sienten. Además, dado que la autoestima varía en función de la satisfacción corporal, esta suele ser muy baja, generando sentimientos de ineficacia y desvalimiento. La adolescente se siente entonces descontenta con su cuerpo y no será extraño que en el campo de la sexualidad, aparezca un elevado temor a reconocer los propios impulsos libidinales, pudiendo incluso llegar a anularlos (Raich, 1998; Rausch \& Bay, 1997).

La desconfianza en los propios recursos también repercute en el ámbito de las interacciones sociales. Disminuye el interés por establecer relaciones íntimas, pues, al parecer, el mantener a los demás a una determinada distancia permite experimentar cierta seguridad. De esta manera, la joven se aísla del entorno e incluso en aquellas pocas oportunidades en las cuales sí interactúa con otras personas, generalmente conocidas, se evidencian limitaciones en la espontaneidad, originándose así un comportamiento inhibido (Raich, 1998; Rausch, 1996). 
En cuanto a la bulimia, las dificultades emocionales son también evidentes. Una vez instalada la patología se acrecienta la inestabilidad emocional, desencadenándose rápidos cambios en el humor que dependerán en general del grado de satisfacción corporal. De igual manera, la joven bulímica presentará una autoestima baja, pues siente que así como ella desvaloriza su cuerpo, los demás responden igual. Su única fuente de satisfacción provendrá del resultado de las dietas; sin embargo, estas tienden a fracasar y a desencadenar atracones (Raich, 1998; Rausch, 1996).

Por otra parte, se evidencia un elevado descontrol en los impulsos que, aunada a la baja tolerancia a la frustración, llevan a algunas pacientes a convertirse en gastadoras compulsivas, mitómanas o cleptómanas (roban comida u otros objetos). Otras incurren en el abuso de sustancias psicoactivas, exhiben una sexualidad desordenada e incluso se autolesionan o cometen gestos suicidas (Vásquez et al., 2004). Por esta razón, alrededor del $20 \%$ de las bulímicas presentan comorbilidad con el trastorno límite de la personalidad (APA, 2002). Las fluctuaciones tan marcadas en el humor repercuten negativamente en el funcionamiento social. Más aún, las interacciones con el medio dependerán de los atracones, que no solo consumen tiempo y dinero sino que la obligan a bloquear contactos satisfactorios con el entorno. Si la joven presenta ya una orientación extrovertida, establecerá vínculos superficiales en los que dependerá de los demás para obtener gratificación. Otras adolescentes, por el contrario, se aíslan de quienes la rodean y con ello se intensifican los grados de preocupación, inquietud y malestar (Rausch, 1996).

Finalmente, sea que se utilice la inanición o los atracones y purgas, es la corporalidad la que termina expresando los propios impulsos y emociones. Por lo tanto, surge la siguiente pregunta: ¿qué otras características presenta la afectividad en aquellas adolescentes que padecen anorexia y bulimia? Para responder dicha interrogante, se plantearon los siguientes objetivos:

a) Identificar las características de los afectos en un grupo de adolescentes diagnosticadas con anorexia nerviosa en función de las variables sociodemográficas y de enfermedad.

b) Identificar las características de los afectos en un grupo de adolescentes diagnosticadas con bulimia nerviosa en función de las variables sociodemográficas y de enfermedad.

c) Generar un perfil de las adolescentes con trastornos alimentarios en función de las variables sociodemográficas y de enfermedad.

d) Comparar las características de afectividad del grupo de adolescentes anoréxicas con el grupo de adolescentes bulímicas. 
MÉTOdO

\section{Participantes}

Participaron en este estudio 35 adolescentes mujeres, asistentes a una clínica de día, 18 de ellas diagnosticadas con anorexia nerviosa y 17 con bulimia nerviosa. El rango de edades oscila entre los 12 y 18 años, siendo el promedio los 15 años (57,1\%). En cuanto al nivel socioeconómico, ${ }^{1} 19$ pertenecían a un nivel socioeconómico alto (54,3\%), diez se ubicaban en el sector medio $(28,6 \%)$ y las ocho restantes pertenecían al estrato bajo (17,1\%). Se incluyeron solo a las pacientes que cursaban la fase inicial del tratamiento y se excluyó a adolescentes diagnosticadas con trastornos de pensamiento o personalidad.

\section{Medición}

- Psicodiagnóstico de Rorschach mediante el sistema comprehensivo del Rorschach.- El psicodiagnóstico de Rorschach es una prueba perceptiva y proyectiva que permite recolectar datos sobre los procesos de la personalidad. No obstante, el Sistema Comprehensivo constituye un método multifacético que, gracias a su alta confiabilidad y validez -para la aplicación, calificación e interpreta-

El nivel socioeconómico fue establecido en base al distrito de residencia, el tipo de colegio al que la joven asistía (estatal o privado y de nivel alto, medio o bajo) y el trabajo que desempeñaban ambos padres. ción de los resultados- otorga a la prueba características psicométricas (Anastasi \& Urbina, 1998; Weiner, 1997).

- Ficha sociodemográfica.- Confeccionada para fines de la investigación, registraba datos generales de la paciente, como la edad y el nivel socioeconómico al cual pertenecía. Además, permitió recoger información relacionada con la enfermedad, tal como el diagnóstico, el tiempo de enfermedad y de tratamiento, detectar si existía historia de consumo de sustancias psicoactivas, de autolesiones e ideación o intento suicida e inicio sexual. Asimismo, exploraba la presencia de conductas oposicionistas, compulsivas y evaluaba el grado de socialización, el ánimo, el nivel de conciencia de enfermedad y el grado de adherencia al tratamiento. Finalmente, permitió recoger datos sobre la familia de la paciente, como las personas con quienes vivían las participantes, la posición ordinal fraternal que ocupaban y el tipo de familia de la cual provenían. Para completar dicho instrumento se utilizaron las historias clínicas y se solicitó la ayuda del personal del departamento de Psicología.

\section{Procedimiento}

En primer lugar, se contactó a la institución a partir de la cual se extrajo la muestra y se planteó solicitar el consentimiento informado a los padres y 
pacientes; sin embargo, dicha solicitud fue negada, pues la institución contaba ya con una autorización interna para este tipo de intervenciones. Por lo tanto, la aplicación del psicodiagnóstico de Rorschach se enmarcó dentro de las evaluaciones iniciales de las pacientes. Posteriormente, se decidió asistir a la clínica en calidad de practicante por cuatro meses a fin de establecer el rapport con las jóvenes, teniendo en cuenta las posibles resistencias. No obstante, diez de las 35 participantes fueron evaluadas sin haberse podido establecer dicho clima de confianza, dado que se informó su pronto retiro de la institución. Durante este tiempo, además, la investigadora realizó observaciones, elaboró talleres grupales dirigidos a las adolescentes e interactuó con ellas y sus familias. Dicho espacio permitió conocer la forma como se perciben a sí mismas, sus miedos, obsesiones y estrategias de afrontamiento, lo cual resultó beneficioso para el posterior análisis de los resultados. Asimismo, se participó en reuniones clínicas en las cuales se recogieron las opiniones y aportes del equipo terapéutico y clínico.

Luego de este periodo, se procedió a la recolección de datos, con lo que se completó la ficha sociodemográfica. Para ello se revisó la historia clínica de cada participante y se conversó con los terapeutas individuales, grupales y familiares. A continuación, se realizaron sesiones de alrededor de una hora en las que se aplicó el psicodiagnósti- co de Rorschach. Conforme las jóvenes elaboraron sus respuestas, estas se anotaron literalmente. Al finalizar cada aplicación se redactaron observaciones relevantes para la posterior interpretación. En seguida se transcribieron las respuestas y se codificaron todos los protocolos bajo los estándares del sistema comprehensivo del Rorschach y se buscó asegurar la confiabilidad de la codificación, para lo cual se seleccionaron al azar tres protocolos de pacientes anoréxicas y otros tres de pacientes bulímicas. Estos fueron entregados a dos jueces, quienes los codificaron independientemente, obteniéndose un nivel de confianza de $85,90 \%$, el que se consideró alto. Posteriormente, se traspasaron los datos al programa RIAP 5.0, software especialmente diseñado para transformar dicha información en cifras numéricas.

En la siguiente etapa, se analizaron las variables que, acorde con lo propuesto por Sendín (2007), se relacionan directamente con el estudio de los afectos y los valores obtenidos en las variables propuestas y fueron procesados haciendo uso del programa estadístico SPSS 17.0. Si bien el estudio se centró en los datos cuantitativos, se incluyó también un análisis cualitativo de las respuestas pues ello permitió ampliar y enriquecer los resultados encontrados.

\section{Resultados}

- Perfil del grupo de estudio.- Las participantes en su mayoría perte- 
necen a familias nucleares $(54,3 \%)$, poseen uno o más hermanos $(82,9 \%)$ y habían sido las últimas en nacer (42,9\%). Destaca en general una actitud sobreprotectora $(54,3 \%)$ por parte de los padres hacia las pacientes. En cuanto al inicio de la enfermedad, más de la mitad de jóvenes anoréxicas $(64,3 \%)$ empezaron su enfermedad entre los 12 y 14 años, mientras que la mayoría de las bulímicas $(66,6 \%)$ presentaban un inicio más tardío, entre los 15 y 18 años. Así, al momento de la investigación, la mayor parte de las participantes $(57,1 \%)$ eran pacientes con más de dos años de enfermedad. Un 74,3\% de las adolescentes con patología alimentaria aún llevaban menos de seis meses en tratamiento y solo en dos casos $(5,7 \%)$ las pacientes aceptaban padecer de un trastorno alimentario. Finalmente, con respecto a los rasgos de personalidad, no se encontró que primara una tendencia.

- Indicadores del psicodiagnóstico de Rorschach por grupos.- Al comparar los dos grupos de estudio, el grupo de bulímicas puntuó más alto que el de anoréxicas en los determinantes de color cromático puro (C) y movimiento inanimado (m), además del contenido de fuego (Fi). Sin embargo, dado que no hubo diferencias significativas en la mayoría de variables, se conformó el grupo de "adolescentes con TCA" representado por 35 sujetos, el mismo que fue comparado con la data proporcionada por Exner (2007).

- Estructura de personalidad en el grupo con TCA.- Todas las evaluadas dieron más de 14 respuestas, considerándose todos los protocolos como válidos (Sendín, 2007). Con respecto al Lambda (L), no existe una tendencia en particular. Sin embargo, doce jóvenes $(34,3 \%)$ obtuvieron un L mayor a 0.99 , ante lo cual Sendín (2007) propone descartarlos. A pesar de ello, se decidió incluirlos, pues el $L$ alto en algunas participantes parece deberse no solo a un estilo defensivo sino también a la presencia de rasgos evitativos en el procesamiento de la realidad. De otra parte, se encontraron diferencias significativas para los determinantes de movimiento animal $(\mathrm{FM}=2.14)$ y movimiento inanimado $(\mathrm{m}=2.17)$, además de la puntuación $\mathrm{D}(\mathrm{D}=-0.87)$ para los 17 años. En cuanto al estilo vivencial (EB), 13 jóvenes eran introversivas (37,1\%), otras $13(37,1 \%)$ utilizaban el estilo ambigual y solo nueve adolescentes $(25,7 \%)$ eran extratensivas. No obstante, resalta la alta rigidez en el uso de los estilos introversivo y extratensivo (EBPer > 1,30).

- Afectividad.- Al analizar el índice de inhabilidad social (CDI), se encontró que a medida que las adolescentes aumentan de edad, los casos de CDI $>3$ también ascienden. En el lado derecho de la experiencia base (eb), se encontraron puntajes más altos 
en los determinantes de color acromático (C') y de sombreado difuso (Y) así como un puntaje menor para el determinante de textura $(\mathrm{T})$ (véase la tabla 1). Con respecto a la responsividad a estímulos emocionales (Afr), solo se encontraron diferencias significativas para los grupos de TCA de 16 y 17 años, quienes puntuaron más bajo de lo esperado (véase la tabla 1) (Exner, 2007). Al estudiarse la proporción forma-color $(\mathrm{FC}: \mathrm{CF}+\mathrm{C})$, primaba una tendencia a regular la expresión del afecto gracias al uso de elementos cognitivos (48,6\%); no obstante, otra parte del grupo (40\%) operaba de la forma contraria, dejándose más bien guiar por sus emociones. Solo cuatro adolescentes no presentaban un estilo definido $(11,4 \%)$.
A pesar de ello, al estudiar la presencia de color cromático puro (C), sí se encontraron diferencias significativas (véase la tabla 1). Finalmente, se exploraron las respuestas de espacio en blanco (S), hallándose un mayor puntaje de lo esperado (véase la tabla 1).

En relación con el porcentaje de respuestas complejas, se obtuvo un promedio de $18,97 \%$, que dista del $25 \%$ esperado para esta etapa (Sendín, 2007). Por otro lado, se encontró que un $41,6 \%$ de las complejas presentan calidad formal menos (FQ-). Destaca también que los contenidos anatómicos y radiográficos solo constituyen un 2,9\%. Finalmente, impresiona haber hallado 10 combinaciones sombreado-sombreado (ShdShd).

Tabla 1

Variables significativas en el cluster de afectividad para adolescentes con TCA

\begin{tabular}{cccccccc}
\hline \multirow{2}{*}{$\begin{array}{c}\text { Variables } \\
\text { Rorschach }\end{array}$} & $\begin{array}{c}\text { Grupo } \\
\text { etario }\end{array}$ & \multicolumn{2}{c}{ TCA } & \multicolumn{2}{c}{ Baremos } & T de & Sig \\
\cline { 3 - 6 } & Media & DS & Media & DS & Student & \\
\hline C' & 12 a 18 años & 3,31 & 2,81 & 1,24 & 1,72 & 4,37 & $0,00^{* *}$ \\
T & 12 a 18 años & 0,14 & 0,35 & 0,98 & 0,35 & $-13,95$ & $0,00^{* *}$ \\
Y & 17 años & 2,00 & 1,51 & 0,61 & 0,96 & 3,56 & $0,00^{* *}$ \\
Afr & 16 años & 0,50 & 0,15 & 0,65 & 0,17 & $-2,92$ & $0,02^{*}$ \\
& 17 años & 0,43 & 0,11 & 0,67 & 0,16 & $-8,70$ & $0,00^{* *}$ \\
C & 12 y 18 años & 1,06 & 1,14 & 0,13 & 0,50 & 4,83 & $0,00^{* *}$ \\
S & 12 a 18 años & 2,77 & 2,29 & 1,47 & 1,76 & 3,36 & $0,02^{*}$ \\
\hline
\end{tabular}

${ }^{*} p<.05$

${ }^{* *} p<.01$ 
- Otros indicadores.- En primer lugar, existen diferencias significativas entre los dos grupos de estudio originales para el determinante de reflejo $(\mathrm{Fr}+\mathrm{rF})$, el cual es mayor en las bulímicas $(\mathrm{X}=1,18)$ que en las anoréxicas $(X=0,33)$. Ya al comparar el grupo de TCA con los valores propuestos por Exner (2007) se encontró que los contenidos anatómicos (An) y radiográficos (Xy) se presentan dentro de los parámetros normales. Sin embargo, las pocas veces que aparecen, vienen asociados a contenidos mórbidos (MOR) o a una calidad formal menos (FQ-).

En segundo término, al analizar algunas variables del cluster de re- laciones interpersonales, se encontraron diferencias significativas. De esta manera, los puntajes de los contenidos agresivos (AG), cooperativos (COP) y de buenas relaciones interpersonales (GHR) son menores a lo esperado; mientras que en la variable de pobres relaciones interpersonales (PHR) las puntuaciones se muestran elevadas (véase la tabla 2).

Con respecto al componente cognitivo, se revisó el índice de trastorno de percepción-pensamiento (PTI) para el total de la muestra, obteniéndose un promedio de 2,09. E1 análisis de las respuestas populares (P) reveló un puntaje menor de lo esperado (véase la tabla 2); sin em-

Tabla 2

Otros indicadores del sistema comprehensivo en adolescentes con TCA

\begin{tabular}{|c|c|c|c|c|c|c|c|}
\hline \multirow{2}{*}{$\begin{array}{l}\text { Variables } \\
\text { Rorschach }\end{array}$} & \multirow{2}{*}{$\begin{array}{l}\text { Grupo } \\
\text { etario }\end{array}$} & \multicolumn{2}{|c|}{ TCA } & \multicolumn{2}{|c|}{ Baremos } & \multirow{2}{*}{$\begin{array}{c}\text { T de } \\
\text { Student }\end{array}$} & \multirow[t]{2}{*}{ Sig } \\
\hline & & Media & DS & Media & DS & & \\
\hline$A G$ & 12 a 18 años & 0,23 & 0,55 & 1,15 & 0,63 & $-9,97$ & $0,00^{* *}$ \\
\hline COP & 12 a 18 años & 0,60 & 0,74 & 1,61 & 0,83 & $-8,20$ & $0,00^{\star *}$ \\
\hline GHR & 12 a 18 años & 3,49 & 1,98 & 5,23 & 2,44 & $-5,22$ & $0,00^{* *}$ \\
\hline \multirow[t]{3}{*}{ PHR } & 12 a 15 años & 6,09 & 305 & 1,30 & 1,16 & 5,21 & $0,00^{* *}$ \\
\hline & 16 años & 4,89 & 3,92 & 1,16 & 1,29 & 2,85 & $0,02^{*}$ \\
\hline & 17 años & 3,60 & 2,64 & 1,53 & 1,46 & 3,04 & $0,01^{*}$ \\
\hline$P$ & 12 a 18 años & 5,17 & 1,79 & 6,30 & 1,25 & $-3,73$ & $0,01^{*}$ \\
\hline S- & 12 a 18 años & 1,43 & 1,42 & 0,41 & 0,66 & 4,24 & $0,00^{\star *}$ \\
\hline
\end{tabular}

${ }^{*} p<.05$

${ }^{* *} p<.01$ 
bargo, un $83,71 \%$ de estas respuestas se asociaron a una calidad formal ordinaria (FQo). Por último, se presentó un elevado uso de respuestas en blanco asociadas a una calidad formal menos (S-) (véase la tabla 2).

- Análisis cualitativo.- A partir del análisis de las respuestas, se observa que experiencias adversas tempranas parecen haber determinado daños en la percepción del sí mismo y de los demás, trayendo consigo repercusiones negativas en la esfera relacional. Los demás son vistos como fuente de escaso soporte afectivo y por lo tanto surgiría la sensación de ser incomprendida y estar sola. En este contexto, aparecería una intensa afectividad disfórica que, al no poder ser canalizada de manera efectiva, originaría una fijación en el cuerpo y en la alimentación.

\section{DISCUSIÓN}

Consolidar la propia identidad y fortalecer los controles afectivos constituyen tareas complejas para cualquier adolescente mujer. Sentirse a gusto con el nuevo cuerpo y la sexualidad así como lograr el control de los impulsos y de las emociones negativas puede llegar a ser difícil. A pesar de ello, la mayoría de adolescentes logra superar estas tareas con éxito. No obstante, algunas otras presentan dificultades para evaluarse a sí mismas y a los demás de manera positiva y, en consecuencia, experimentan una afectividad muy distinta. Este es el caso de las jóvenes que desarrollan trastornos alimentarios como la anorexia y la bulimia.

La mayoría de estas pacientes no logra desarrollar estrategias eficaces para procesar estímulos internos y externos. En algunas oportunidades evitan complicarse con la estimulación, mientras que en otras pueden verse abrumadas por la excesiva recopilación de información. Igualmente, oscilan entre apelar a la lógica o a las emociones para el procesamiento de la información. Si bien esto es esperable para la población adolescente que aún pone a prueba diferentes estilos (Exner, 2007), conforme las jóvenes con patología alimentaria avanzan en edad, este estilo inseguro y dubitativo se fortalece en vez de debilitarse, prolongándose la orientación infantil que disminuye el ajuste y la eficacia de las conductas. Aquellas pocas que, por el contrario, sí desarrollan un estilo particular para enfrentarse al medio, lo utilizan de manera rígida, no pudiendo adaptarse a las exigencias de las diversas situaciones.

Por otro lado, estas adolescentes presentan un pobre reconocimiento del hambre, expresión básica del instinto de supervivencia que, al ser negada o alterada, denota una intensa disociación con el propio cuerpo y que levanta interrogantes sobre las causas y objetivos de dicha alteración.

Además de la falta de estilos definidos y la negación del hambre como 
características estables de la personalidad, las adolescentes presentan una elevada ansiedad situacional, que se acentúa especialmente en el grupo de bulímicas. Dicho estado emocional surge del gran temor que genera el entrar a un tratamiento donde se les fuerza a alimentarse con regularidad, lo cual supone finalmente ganar peso.

Se hace evidente entonces que los trastornos alimentarios no solo generan déficits físicos, afectivos y sociales, sino que tiñen la estructura de personalidad y obstaculizan el crecimiento psicológico. Progresivamente, limitan los recursos disponibles, haciendo que las demandas del medio sean percibidas como superiores a las propias capacidades. En ese sentido, estos desórdenes van más allá del cuerpo, de la comida o del peso.

En relación con la afectividad, es esperable cierta disforia, pues la adolescencia implica dejar atrás el cuerpo y la identidad infantil, así como la protección de los padres. En ese sentido, puede generar duelos, reeditar angustias y despertar ansiedades latentes (De las Casas, 2008). No obstante, las jóvenes con TCA experimentan mayor tristeza y miedo que sus pares así como sentimientos de soledad y abandono, además de la ansiedad ya mencionada. Adicionalmente, sus intentos por autoafirmarse son tan elevados que determinan una actitud oposicionista debajo de la cual subyacen agresividad y sentimientos de hostilidad.
La población adolescente normal suele internalizar parte de estos afectos; sin embargo, encontramos que en las pacientes con TCA la constricción afectiva sería elevada, pudiendo inducir la aparición de alteraciones psicosomáticas (Sendín, 2007). De manera crónica, estas pacientes tienden a reprimir la expresión de sus afectos, dirigiendo las emociones displacenteras hacia su interior. Al parecer, dicha estrategia permitiría compensar la labilidad afectiva, neutralizando el riesgo del descontrol. A pesar de ello, los afectos dolorosos no desaparecen, sino que se reprimen, aumentando más bien la tensión y el malestar. Entonces, el sufrimiento psicológico sobrepasaría las defensas habituales y el cuerpo se convertiría en el canal a través del cual toda esta angustia podría ser expresada (McDougall, 1989; Zusman, 2009).

Por otro lado, las adolescentes con TCA exhiben una menor necesidad de contacto emocional (T ausente) que sus pares. Si bien dicho hallazgo debe interpretarse a la luz de las influencias culturales, pues se sabe que algunas poblaciones latinoamericanas tenderían a puntuar más bajo en el determinante de textura (T) (Dana, 2005), diversos indicadores en la investigación hacen suponer que el contacto afectivo genera confusión o malestar en vez de gratificación, pudiendo esto influir en la actual distancia emocional.

Siguiendo esta línea, se pudo constatar que entre los 12 y 15 años existe 
un interés promedio por procesar estímulos afectivos; sin embargo, hacia el final de la adolescencia -a los 16 y 17 años- esta tendencia decae, evidenciándose que con el pasar del tiempo, las jóvenes con TCA van apartándose de la estimulación afectiva. Prefieren no implicarse en situaciones afectivas, pues estas podrían ser disparadoras del descontrol, especialmente en el caso de las bulímicas. De ahí que sus relaciones con los demás sean superficiales y distantes en el nivel emocional, lo que repercute en su desarrollo, pues, a su vez, pierden intercambios vitales para la formación de una identidad social saludable.

La constricción y la evitación son usadas como medios para mantenerse alejadas de esta estimulación, pero ambas estrategias tienden a fallar en situaciones de estrés, en las cuales tanto anoréxicas como bulímicas pueden perder el control y descargar sus emociones de manera brusca. Al respecto, este hallazgo resulta especialmente significativo para el caso de la anorexia nerviosa, pues se suele asumir que estas pacientes presentan rasgos obsesivos gracias a los cuales son capaces de manejar sus funciones fisiológicas y la expresión de sus afectos (Rausch, 1996). No obstante, se ha podido comprobar que el control absoluto es imposible, y hay ocasiones en las cuales las defensas no son suficientes y entonces todo el afecto reprimido es expulsado sin modulación alguna.

Ante la presencia de estilos tan particulares de manejar la afectividad, se decidió examinar las respuestas com- plejas, incluir algunas otras variables de estudio y complementar los resultados con un análisis cualitativo del Rorschach. Allí se encontró que experiencias emocionales extremadamente penosas en el pasado (respuestas de sombreado-sombreado, antecedentes de abuso sexual, maltrato psicológico, límites familiares difusos, entre otros) habrían determinado que las jóvenes de la muestra presenten un yo débil y poco integrado, incapaz de afrontar las demandas propias de la adolescencia. A la vez, estas mismas vivencias habrían generado representaciones distorsionadas de los otros, en las cuales los demás, y en especial las figuras masculinas, son vistas como fuentes de escaso soporte. En consecuencia, es posible que ya antes de entrar a la pubertad, estas jóvenes hayan carecido de los recursos personales y sociales, tan necesarios para afrontar las diversas tareas que trae consigo esta etapa.

Al llegar a la adolescencia, la sensación de vulnerabilidad y desamparo habrían aumentado, siendo fácil el verse abrumada por los afectos displacenteros. Estos, al ser intensos, impactan en la esfera cognitiva, limitando el rendimiento intelectual y determinando un funcionamiento menos elaborado. Adicionalmente, cuando la afectividad se combina con la ideación, el procesamiento de la realidad se ve distorsionado, dando como resultado percepciones idiosincráticas. 
Conforme la joven crece, la afectividad disfórica, los sesgos cognitivos y la pobre representación de los otros cobran fuerza, originando mayores dificultades sociales. En ese sentido, el aumento en el CDI positivo hacia el final de la adolescencia comprobaría cómo ya a los 17 años existen serios déficits relacionales en los cuales el encuentro con los demás no brinda aspectos positivos ni negativos, constituyendo estas figuras incapaces de otorgar el soporte afectivo que ellas necesitan.

Al no disponer de recursos ni de soportes externos que les permitan lidiar con las emociones negativas, no resulta extraño que todo el vacío interior, la tristeza, la angustia y la rabia se dirijan hacia el interior, siendo el cuerpo deprivado la única forma de expresarse. Sin embargo, la preocupación por el cuerpo que presentan las jóvenes con TCA se encuentra dentro del promedio, evidenciando una disociación de lo que anatómicamente es evidente. Ello, al parecer, responde a que las veces en las que se piensa en la propia corporalidad aparecen distorsiones y sensaciones displacenteras.

Por otra parte, este hallazgo refuerza aquellas propuestas teóricas que consideran que la problemática en los trastornos alimentarios va más allá de la corporalidad. El conflicto entonces no es con la imagen corporal, con el peso o con la comida, sino que el cuerpo solo sirve como medio de expresión de toda la angustia interna.
Al ser incapaces de representar y dar sentido a sus experiencias, las adolescentes con TCA vuelven a etapas anteriores en las que el cuerpo servía como el único medio de sentir y expresar. En ese sentido, si desde la infancia no se ha contado con adultos que brinden soporte y ayuden a elaborar las propias experiencias, será difícil que luego, durante la adolescencia, las jóvenes puedan acceder a las representaciones de la palabra para reconocer y expresar sus sentimientos (Dorado de Lisondo, 2008). Entonces, dado que no se puede usar el lenguaje, será el cuerpo el que exprese lo que la mente no puede (McDougall, 1989).

Finalmente, el cuerpo infantil les brindaría a estas jóvenes la ilusión de ser todavía niñas alejadas de los conflictos y exigencias del mundo adulto que resultan imposibles de afrontar. Ello incluiría también el ámbito de la sexualidad, pues cabe recordar que en la adolescencia, el cuerpo evoluciona a uno sexualmente maduro y con ello queda conformada parte de la identidad femenina. De esta manera, pareciera como que estas adolescentes se niegan a crecer, rechazan el cuerpo femenino y la sexualidad adulta, y en este contexto, es el adelgazamiento el que les permite conservar ese status infantil (De las Casas, 2008; Zusman, 2009).

En conclusión, es cierto que el medio sociocultural influye en la construcción de la identidad y en la afectividad 
femenina al promover cuerpos andróginos y tubulares; sin embargo, el papel que cumple la familia es aún más importante. Así, el haber crecido en un entorno confuso, que no favorece la autonomía, que desaprueba el crecimiento físico, y que sobre todo, no es capaz de contener y brindar el soporte afectivo necesario para construir una identidad segura y coherente, impide que estas jóvenes desarrollen suficientes recursos afectivos para afrontar todas las demandas de la adolescencia. Por lo tanto, al entrar a esta etapa tan crítica, toda la angustia y el sufrimiento que no pueden ser expresadas mediante el discurso se terminan poniendo en acciones. Entonces, se regresiona a etapas primitivas en las que la mente y el cuerpo constituían una unidad indivisible, y por tanto, las vivencias psíquicas solo podían expresarse a través de la corporalidad (Zusman de Arbiser, 1999).

\section{CONCLUSIONES}

Al estudiar la afectividad en el grupo de jóvenes con trastornos alimenticios, se encontraron diferencias significativas en cuanto a los determinantes de color acromático (C'), color cromático puro (C), sombreado difuso (Y) y textura (T), así como para las respuestas de espacio en blanco (S). La responsividad a estímulos emocionales (Afr) solo presentaba diferencias significativas para los grupos de TCA de 16 y 17. El porcentaje de respuestas complejas se en- contraba por debajo del promedio y en $41,6 \%$ de los casos presentaban calidad formal menos (FQ-). Destacó también que los contenidos anatómicos y radiográficos solo constituyeran un 2,9\%. Finalmente, impresionó haber hallado 10 combinaciones sombreado-sombreado (Shd-Shd).

\section{REFERENCIAS}

Anastasi, A., \& Urbina, S. (1998). Tests psicológicos (7. edición). México D. F.: Prentice Hall.

Asociación Psiquiátrica Americana (2002). Manual diagnóstico y estadístico de los trastornos mentales $I V$ (versión revisada). Barcelona: Masson.

Barbarich, N., Kaye, W., \& Jimerson, D. (2003). Neurotransmitter and imaging studies in anorexia nervosa: New targets for treatment. Current Drugs Targets - CNS \& Neurological Disorders, 2, 61-72.

Dana, R. (2005). Multicultural assessment: Principles, assessment and examples. New Jersey: Lawrence Erlbaum.

De las Casas, P. (2008). Un cuerpo sin sombras. Anorexia y bulimia: Una relectura en Freud. Lima: Universidad Nacional Mayor de San Marcos, Fondo Editorial.

Dorado de Lisondo, A. (2008). Las patologías del vacío. Transiciones, 13, 133-160. 
Erikson, E. (1979). Sociedad y adolescencia. México D. F.: Siglo XXI.

Exner, J. (2007). Manual de codificación del Rorschach para el sistema comprehensivo (5. ${ }^{\text {a }}$ edición). Madrid: Psimática.

Freud, A. (1971). El yo y los mecanismos de defensa (5. edición). Buenos Aires: Paidós.

Hevia, J. (2003). Imagen y realidad del cuerpo en el mundo de hoy. Persona, 6, 53-73.

Horrocks, J. (1986). Psicología de la adolescencia. México, D. F.: Trillas.

Kirzman, D. (2002). El enemigo en el espejo: De la insatisfacción corporal al trastorno alimentario. Madrid: TEA Ediciones.

Kogan, L. (2003). La construcción social de los cuerpos o los cuerpos del capitalismo tardío. Persona 6, 11-21.

McDougall, J. (1989). Teatros del cuerpo. Madrid: Julián Yebenés.

Muuss, R. (1974). Teorías de la adolescencia. Buenos Aires: Paidós.

Papalia, D., Olds, S., \& Feldman, R. (2009). Psicología del desarrollo: De la infancia a la adolescencia (11. a edición). Bogotá: McGraw-Hill.

Polivy, J., \& Herman, P. (2002). Causes of eating disorders. Annual Review of Psychology, 53 (1), 187-213.
Raich, R. (1998). Anorexia y bulimia: Trastornos alimentarios. Madrid: Ediciones Pirámide.

Raich, R. (2001). Imagen corporal: Conocer y valorar el propio cuerpo. Madrid: Ediciones Pirámide.

Rausch, C. (1996). La esclavitud de las dietas: Guía para reconocer y encarar un trastorno alimentario. Buenos Aires: Paidós.

Rausch, C., \& Bay, L. (1997). Anorexia y bulimia. Amenazas a la autonomía. Buenos Aires: Paidós.

Reusche, R. (1999). La adolescencia: Desafio y decisiones. Lima: Universidad Femenina del Sagrado Corazón.

Sendín, M. (2007). Manual de interpretación del Rorschach para el sistema comprehensivo (3. a edición). Madrid: Psimática.

Vásquez, F., Calizaya, R., Campos, G., Alfaro, O., Contreras, A., Nicolás, Y., et al. (2004). Los vómitos que aproximan a la muerte. El complejo problema de la bulimia y el intento de autoeliminarse: Revista de Neuropsiquiatría, 7, 249-256.

Weiner, I. (1997). Current status of the Rorschach Inkblot Method. Journal of Personality Assessment, 68 (1), 5-19. 
Zusman, L. (2009). Los desórdenes de la conducta alimentaria: Anorexia y bulimia. Lima: Pontificia Universidad Católica del Perú, Fondo Editorial.
Zusman de Arbiser, S. (1999). Sobreadaptación y enfermedad psicosomática en niños y adolescentes: Distintos abordajes terapéuticos. Revista de Psicoanálisis, 56, (2), 335-3. 